\title{
A Vizing-type result for semi-total domination
}

\author{
John Asplund \\ Department of Technology and Mathematics \\ Dalton State College \\ Dalton, GA 30720, USA \\ jasplund@daltonstate.edu \\ Randy Davila \\ Department of Pure and Applied Mathematics \\ University of Johannesburg \\ Auckland Park 2006, South Africa \\ Department of Mathematics and Statistics \\ University of Houston-Downtown \\ Houston, TX 77002, USA \\ Email: davilar@uhd.edu \\ Elliot Krop \\ Department of Mathematics \\ Clayton State University \\ Morrow, GA 30260, USA \\ Email: elliotkrop@clayton.edu
}

\begin{abstract}
A set of vertices $S$ in a simple isolate-free graph $G$ is a semi-total dominating set of $G$ if it is a dominating set of $G$ and every vertex of $S$ is within distance 2 or less with another vertex of $S$. The semi-total domination number of $G$, denoted by $\gamma_{t 2}(G)$, is the minimum cardinality of a semi-total dominating set of $G$. In this paper we study semi-total domination of Cartesian products of graphs. Our main result establishes that for any graphs $G$ and $H, \gamma_{t 2}(G \square H) \geq \frac{1}{3} \gamma_{t 2}(G) \gamma_{t 2}(H)$.
\end{abstract}

Keywords: Cartesian products, total domination number, semi-total domination number.

AMS subject classification: $05 \mathrm{C} 69$ 


\section{Introduction}

In this paper we study bounds on a recently introduced domination invariant applied to Cartesian products of graphs. At its core, our work is motivated by the longstanding conjecture of V.G. Vizing [17] on the domination of product graphs, which states that for any graphs $G$ and $H, \gamma(G \square H) \geq \gamma(G) \gamma(H)$. Here, $\gamma(G)$ is the domination number of $G$, which is the minimum size of a set $D$ of vertices so that every vertex not in $D$ is adjacent to some vertex in $D$, and $\square$ is the Cartesian product of graphs. The breakthrough "double-projection" result of Clark and Suen [5] gave the first Vizingtype bound of $\gamma(G \square H) \geq \frac{1}{2} \gamma(G) \gamma(H)$. Recently, Brešar [1] improved this bound to $\gamma(G \square H) \geq \frac{(2 \gamma(G)-\rho(G)) \gamma(H)}{3}$, where $\rho(G)$ is the two-packing number of $G$. For more on attempts to solve Vizing's conjecture over more than five decades since it was stated, see the survey [2].

Over the years, due to the unyielding nature of the conjecture, devotees have used offshoots of the domination number to attempt Vizing-type inequalities, in hopes of better understanding the difficulties of the original problem. For example, Brešar, Henning, and Rall [4] defined the paired and rainbow domination numbers, and Henning and Rall 12. conjectured a Vizing-type inequality for total domination. This last conjecture was proved by Ho [14, who showed that for any graphs $G$ and $H, \gamma_{t}(G \square H) \geq \frac{1}{2} \gamma_{t}(G) \gamma_{t}(H)$. In this result, $\gamma_{t}(G)$ is the total domination number of $G$, which is the minimum size of a set $T$ of vertices so that every vertex of $G$ is adjacent to some vertex in $T$. A sharp example was given in [12] and the characterization of pairs of graphs attaining equality is an active problem, see [3] and [15].

Since the difference between a totally dominating set and a dominating set is that every vertex in a totally dominating set must be adjacent to some other vertex in that set, while this rule does not have to hold in a dominating set, we find it instructive to consider Vizing-type inequalities for domination invariants that share properties with both domination and total domination. That is, we want to consider some domination function in between domination and total domination. Such a function, first investigated by Goddard, Henning, and McPillan [6], is the semi-total domination number of $G$, $\gamma_{t 2}(G)$, which is the minimum size of a set of vertices $S$ in $G$, so that every vertex of $S$ is of distance at most 2 to some other vertex of $S$, and every vertex not in $S$ is adjacent to a vertex in $S$. Although introduced only a few years ago, this function has seen much recent attention, see [8, 9, 10, 11, 16, 18,

Although we cannot prove it, we believe that $\gamma_{t 2}(G \square H) \geq \frac{1}{2} \gamma_{t 2}(G) \gamma_{t 2}(H)$ for any graphs $G$ and $H$. Our result depends on the method of Clark and Suen [5] and requires more careful analysis of semi-total dominating sets. We show that for any graphs $G$ and $H, \gamma_{t 2}(G \square H) \geq \frac{1}{3} \gamma_{t 2}(G) \gamma_{t 2}(H)$.

Definitions and Notation. For notation and graph terminology, we will typically follow [13. Throughout this paper, all graphs will be considered undirected, simple, connected, and finite. Specifically, let $G$ be a graph with vertex set $V=V(G)$ and edge 
set $E=E(G)$. Two vertices $v, w \in V$ are neighbors, or adjacent, if $v w \in E$. The open neighborhood of $v \in V$, is the set of neighbors of $v$, denoted $N_{G}(v)$, whereas the closed neighborhood is $N_{G}[v]=N_{G}(v) \cup\{v\}$. The open neighborhood of $S \subseteq V$ is the set of all neighbors of vertices in $S$, denoted $N_{G}(S)$, whereas the closed neighborhood of $S$ is $N_{G}[S]=N_{G}(S) \cup S$. The distance between two vertices $v, w \in V$ is the length of a shortest $(v, w)$-path in $G$, and is denoted by $d_{G}(v, w)$. The Cartesian product of two graphs $G\left(V_{1}, E_{1}\right)$ and $H\left(V_{2}, E_{2}\right)$, denoted by $G \square H$, is a graph with vertex set $V_{1} \times V_{2}$ and edge set $E(G \square H)=\left\{\left(\left(u_{1}, v_{1}\right),\left(u_{2}, v_{2}\right)\right): v_{1}=v_{2}\right.$ and $\left(u_{1}, u_{2}\right) \in E_{1}$, or $u_{1}=$ $u_{2}$ and $\left.\left(v_{1}, v_{2}\right) \in E_{2}\right\}$.

A subset of vertices $S \subseteq V(G)$ is called a semi-total dominating set if $N[S]=V(G)$ and for any vertex $u \in S$, there exists a vertex $v \in S$ so that $d(u, v) \leq 2$. The semi-total domination number of $G$, written $\gamma_{t 2}(G)$, is the size of a minimum semi-total dominating set of $G$. A 2-packing is a subset of vertices $T$ of $G$ so that every pair of vertices in $T$ is of distance at least 3. The size of a maximum 2-packing of $G$ is called the 2-packing number, and is written $\rho(G)$.

We will also make use the standard notation $[k]=\{1, \ldots, k\}$, and for two vertices $u, v$, we write $u \sim v$ to indicate that $u$ is adjacent to $v$.

\section{Main Results}

In this section we provide our main results. We begin by establishing a Vizing's-type result which makes use of the 2-packing number.

Theorem 1 For any isolate-free graphs $G$ and $H$,

$$
\gamma_{t 2}(G \square H) \geq \rho(G) \gamma_{t 2}(H) .
$$

Proof. Without loss of generality, we assume $G$ is a $(\rho, \gamma)$-graph (where $\rho=\gamma$ ), and let $\left\{v_{1}, \ldots, v_{\rho(G)}\right\}$ be a maximum 2-packing of $G$. Since each vertex from our packing is distance at least 3 from any other vertex of our packing, we observe that for $i=$ $1, \ldots, \rho(G)$, the closed neighborhoods $N\left[v_{i}\right]$ are pairwise disjoint. Let $\left\{V_{1}, \ldots, V_{\rho(G)}\right\}$ be a partition of $V(G)$ such that $N\left[v_{i}\right] \subseteq V_{i}$. Let $D$ be a $\gamma_{t 2}(G \square H)$-set. For $i=1, \ldots, \rho(G)$, let $D_{i}=D \cap\left(V_{i} \times V(H)\right)$, and let $H_{i}=\left\{v_{i}\right\} \times V(H)$. Further, let $S_{i}$ be a minimum set of vertices in $G \square H$ that semi-totally dominates $H_{i}$, and contains as many vertices in $H_{i}$ as possible. Then, $S_{i} \subseteq V_{i} \times V(H)$. Next suppose that $S_{i}$ contains a vertex $x$ such that $x$ is not in $H_{i}$. Then, $x$ is the unique vertex which semi-totally dominates $x^{\prime}$, for some $x^{\prime} \in H_{i}$. Since $x^{\prime}$ has neighbors, all of which are dominates by vertices in $S_{i}$, if we replace $x$ by $x^{\prime}$ in $S_{i}$, we see that $S_{i}$ is still semi-total dominating (Since $x^{\prime}$ is at distance at least 2 from a vertex which dominates one of its neighbors). Moreover, we have found a set of vertices from $G \square H$ that semi-totally dominates $H_{i}$ and contains more vertices in $H_{i}$ than does $S_{i}$, a contradiction. Hence, we have $S_{i} \subseteq H_{i}$, and so $S_{i}$ is 
a semi-total dominating set of the copy of $H$ in $G \square H$ induced by the set $H_{i}$. Since $D_{i}$ semi-totally dominates $\left\{v_{i}\right\} \times V(H),\left|D_{i}\right| \geq\left|S_{i}\right|$. Thus,

$$
\gamma_{t 2}(G \square H) \geq \sum_{i=1}^{\rho(G)}\left|S_{i}\right| \geq \sum_{i=1}^{\rho(G)} \gamma_{t 2}(H)=\rho(G) \gamma_{t 2}(H) .
$$

Next, we prove a Vizing's type result which relies only on the semi-total domination number. We do this by partitioning minimum semi-total dominating sets into parts that are and are not totally dominating. Notice that for any graph $G$, if $U=\left\{u_{1}, \ldots, u_{k}\right\}$ is a minimum semi-total dominating set of $G$, then $U$ can be separated into two sets, $X$ and $Y$, where $X$ is the set of vertices of $U$ which are adjacent to at least one other vertex of $U$, and $Y=U \backslash X$. We call such sets $X$, allied and such sets $Y$, free.

For any graph $G$, consider the set of minimum semi-total dominating sets of vertices, $\left\{U_{1}, \ldots, U_{k}\right\}$, and for $1 \leq i \leq k$ let $X_{i}$ and $Y_{i}$ be partitions of $U_{i}$ into allied and free sets, respectively. We call $U_{i}$ so that $\left|X_{i}\right|$ is of maximum size for $1 \leq i \leq k$ a maximum allied semi-total dominating set of $G$, the partition $\left\{X_{i}, Y_{i}\right\}$ a maximum allied partition of $G$, the set $X_{i}$ a maximum allied set of $G$, and the set $Y_{i}$ a minimum free set of $G$.

For any maximum allied partition of $G,\{X, Y\}$, let $x(G)=|X|$ and $y(G)=|Y|$.

Theorem 2 For any isolate-free graphs $G$ and $H$,

$$
\gamma_{t 2}(G \square H) \geq \frac{1}{3} \gamma_{t 2}(G) \gamma_{t 2}(H)
$$

Proof. Let $D$ be a minimum semi-total dominating set of $G \square H$. Let $k=\gamma_{t 2}(G)$ and $U=\left\{u_{1}, \ldots, u_{k}\right\}$ be a maximum allied semi-total dominating set of $G$ with maximum allied partition $\{X, Y\}$. Suppose $X=\left\{u_{1}, \ldots, u_{\ell}\right\}$ and $Y=\left\{u_{\ell+1}, \ldots, u_{\ell+m}\right\}$.

Form a partition $\left\{\pi_{1}, \ldots, \pi_{\ell}, \pi_{\ell+1}, \ldots, \pi_{\ell+m}\right\}$ of $V(G)$ where $\pi_{i} \subseteq N\left(u_{i}\right)$ and $x \in \pi_{i}$ implies $x$ is adjacent to $u_{i}$ for $1 \leq i \leq \ell, \pi_{j} \subseteq N\left[u_{j}\right]$ and $x \in \pi_{j}$ implies $x$ is adjacent to $u_{j}$ for $\ell+1 \leq j \leq \ell+m$. Furthermore, we define this partition to have the property that if $u_{i} \in X$ and $u_{j} \in Y$ so that $d\left(u_{i}, u_{j}\right)=2$, then $N\left(u_{i}\right) \cap N\left(u_{j}\right) \cap \pi_{j}=\emptyset$. That is, for any vertex $u_{j}$ of $Y$ which is of distance 2 to some vertex of $X$, there exists a vertex $u$ which is adjacent to $u_{j}$ and to a vertex of $X$, and $u$ belongs to $\pi_{i}$ for some $i \in[\ell]$.

Let $D_{i}=\left(\pi_{i} \times V(H)\right) \cap D$. Let $P_{i}=\left\{v:(u, v) \in D_{i}\right.$ for some $\left.u \in \pi_{i}\right\}$, which are the projections of $D_{i}$ onto $H$. We call vertices of $V(H)$ missing, if they are not dominated from $P_{i}$ and write $M_{i}=V(H)-N_{H}\left[P_{i}\right]$. Vertices of $P_{i}$ which are of distance at most 2 to some other vertex of $P_{i}$ or $M_{i}$ we call covered and write $Q_{i}=\left\{v \in P_{i}: \exists w \in\right.$ $P_{i} \cup M_{i}$ such that $\left.0<d(v, w) \leq 2\right\}$. Vertices of $P_{i}$ of distance at least 3 to other vertices of $P_{i}$ or $M_{i}$ we call uncovered and write $R_{i}=\left\{v \in P_{i}: \forall w \in\left(P_{i} \cup M_{i}\right) \backslash\{v\}, d(v, w) \geq 3\right\}$.

For $v \in V(H)$, let

$$
D^{v}=D \cap(V(G) \times\{v\})=\{(u, v) \in D: u \in V(G)\}
$$


and $C$ be a subset of $\{1, \ldots, k\} \times V(H)$ given by

$$
C=\left\{(i, v): \pi_{i} \times\{v\} \subseteq N_{G \square H}\left(D^{v}\right) \text { or } v \in R_{i}\right\} .
$$

Let $N=|C|$. We will bound $N$ from above by considering the following.

$$
\begin{aligned}
& \mathcal{L}_{i}=\{(i, v) \in C: v \in V(H)\}, \\
& \mathcal{R}^{v}=\{(i, v) \in C: 1 \leq i \leq k\} .
\end{aligned}
$$

These definitions are well-known as they appeared in the seminal work [5], nonetheless, we would like to remind the reader of their interpretation. The set $C$ is a double indexing set, which indicates where you have cells that are either horizontally dominated or dominated by vertices of $R_{i}$. A cell is just a copy of $\pi_{i}$ for some $i$, at some height $v \in V(H)$. We represent $G$ along the horizontal axis of the Cartesian product and $H$ along the vertical. Thus, horizontally dominated cells are precisely, $\pi_{i} \times\{v\}$ which is contained in $N_{G \square H}\left(D^{v}\right)$. Now, $L_{i}$ are elements of $C$ with a fixed $i$ and $R^{v}$ are elements of $C$ along a fixed $v$.

Since counting vertices vertically and horizontally produces the same amount, we have

$$
N=\sum_{i=1}^{k}\left|\mathcal{L}_{i}\right|=\sum_{v \in V(H)}\left|\mathcal{R}^{v}\right| .
$$

Notice that if $v \in M_{i}$, then the vertices in $\pi_{i} \times\{v\}$ which are not in $D^{v}$ must be adjacent to the vertices in $D^{v}$ since $D$ is a semi-total dominating set of $G \square H$. Furthermore, the vertices of $R_{i}$ are counted in $\mathcal{L}_{i}$. This means that $\left|\mathcal{L}_{i}\right| \geq\left|M_{i}\right|+\left|R_{i}\right|$. Hence we obtain the following lower bound for $N$,

$$
N \geq \sum_{i=1}^{k}\left(\left|M_{i}\right|+\left|R_{i}\right|\right)
$$

To find an upper bound on the above quantity, we bound the size of $\mathcal{R}^{v}$.

Claim 1 For any $v \in V(H),\left|\mathcal{R}^{v}\right| \leq 2\left|D^{v}\right|$.

Proof. Suppose $\left|\mathcal{R}^{v}\right|>2\left|D^{v}\right|$ for some $v \in V(H)$. For $(i, v) \in \mathcal{R}^{v}$, by definition, $\pi_{i} \times\{v\} \subseteq N_{G \square H}\left(D^{v}\right)$ or $v \in R_{i}$.

In what follows, we construct a semi-total dominating set $T$ of $G$.

In the first case, if $\pi_{i} \times\{v\} \subseteq N_{G \square H}\left(D^{v}\right)$, we note that if some vertex $x \in \pi_{i}$, then $x$ is adjacent to vertices in $B^{v}$ where $B^{v}$ is the projection of $D^{v}$ onto $G$. 
Subcase 1. Suppose $u \in B^{v}$. If $u \in \pi_{i}$ such that $(i, v) \notin \mathcal{R}^{v}, u \neq u_{i}$ and $1 \leq i \leq \ell+m$, then $u \in N\left(u_{i}\right)$. If $u \in \pi_{i}$ such that $(i, v) \in \mathcal{R}^{v}$, then there exists $\left(u^{\prime}, v\right) \in B^{v}$ such that $u \in N\left(u^{\prime}\right)$. If $u \in \pi_{i}$ such that $(i, v) \notin \mathcal{R}^{v}, u=u_{i}$ for some $\ell+1 \leq i \leq \ell+m$, then notice that we can find a vertex $x_{i}$ which is a neighbor of $u_{i}$ in $\pi_{i}$. Note that $x_{i}$ need not be a member of $B^{v}$, but simply a neighbor of $u_{i}$. Select one such vertex $x_{i}$ for every such $u$, and let $A$ be the set of these vertices $x_{i}$. Thus, $B^{v} \subseteq T, A:=\left\{u_{i}:(i, v) \notin \mathcal{R}^{v}, u_{i} \notin\right.$ $\left.B^{v}, 1 \leq i \leq \ell+m\right\} \subseteq T$, and $\left\{x_{i}:(i, v) \notin \mathcal{R}^{v}, x_{i} \sim u\right.$ for some $\left.u \in U \cap B^{v}\right\} \subseteq T$.

Subcase 2. Suppose $u \in\left\{u_{i}:(i, v) \notin \mathcal{R}^{v}, 1 \leq i \leq \ell\right\}$. If $u \in \pi_{j}$ such that $(j, v) \notin \mathcal{R}^{v}$, then $u \in N\left(u_{j}\right)$. If $u \in \pi_{j}$ such that $(j, v) \in \mathcal{R}^{v}$, then there exists $\left(u^{\prime}, v\right) \in B^{v}$ such that $u \in N\left(u^{\prime}\right)$. Thus, in this subcase, $u$ is adjacent either to a vertex of $B^{v}$ or a vertex $u_{j}$. There are no new vertices that need to be added to $T$.

Subcase 3. Suppose $u \in\left\{u_{i}:(i, v) \notin \mathcal{R}^{v}, \ell+1 \leq i \leq \ell+m\right\}$. Suppose $u$ is of distance 2 to some vertex $u_{j} \in X$. By the definition of the partition, there exists some vertex $w$ adjacent to $u$ and $u_{j}$, so that $w \in \pi_{j^{\prime}}$ for some $j^{\prime} \in[\ell]$. If $\left(j^{\prime}, v\right) \in \mathcal{R}^{v}$, then there exists $u^{\prime} \in B^{v}$ so that $u^{\prime} \sim w \sim u$, which means that $u$ is of distance at least 2 to some vertex of $B^{v}$. Since $T$ contains $B^{v}$, these vertices are already distance 2 from another vertex in $T$.

We are left to consider the case when $u$ is of distance at least 3 to any vertex of $X$. Since $U$ is a minimum semi-total dominating set of $G$, there exists some vertex $u_{j} \in Y$, so that $d\left(u, u_{j}\right)=2$. If $(j, v) \notin \mathcal{R}^{v}$, these vertices are already in $T$ so no action needs to be taken.

If $(j, v) \in \mathcal{R}^{v}$, then there exists some vertex $u^{\prime} \in B^{v}$ so that $u^{\prime} \sim u_{j}$. We will select $u_{j}$ and place it in $T$ to make $T$ a semi-total dominating set of $G$. Notice that in this case, the number of such vertices $u_{j}$ is at most equal to $\left|D^{v}\right|$. Let $S$ be the set of such vertices $u_{j}$, which are of distance 2 to a vertex $u \in Y$ and at least of distance 3 to any vertex of $X$. Then $S$ will be a subset of the set $T$. This finishes Subcase 3 .

In the second case, if $v \in R_{i}$, then since $D$ is a semi-total dominating set, there is some vertex $(u, v) \in\left(\pi_{i} \times\{v\}\right) \cap D^{v}$ and $(w, v) \in\left(\pi_{j} \times\{v\}\right) \cap D^{v}$, for some $j \in[k]$, so that $(u, v)$ is at most distance 2 from $(w, v)$.

Putting these cases together, we have the following disjoint union of sets

$$
\begin{gathered}
T=B^{v} \cup\left\{u_{i}:(i, v) \notin \mathcal{R}^{v}, 1 \leq i \leq \ell\right\} \cup\left\{u_{i}:(i, v) \notin \mathcal{R}^{v}, u_{i} \notin B^{v}, \ell+1 \leq i \leq \ell+m\right\} \\
\cup A \cup S
\end{gathered}
$$

To show $T$ is a semi-total dominating set of $G$, it is enough to show that $T$ is a dominating set, since we showed in each subcase of the first case, and in the second case, that every vertex of $T$ is of distance at most 2 to some other vertex of $T$. If a vertex $u$ is contained in $\pi_{i}$ for $(i, v) \in \mathcal{R}^{v}$, then $u$ is dominated by some vertex of $B^{v}$. If $(i, v) \notin \mathcal{R}^{v}$, then $u$ is dominated either by $\left\{u_{i}:(i, v) \notin \mathcal{R}^{v}, 1 \leq i \leq \ell\right\}$, or $\left\{u_{i}:(i, v) \notin \mathcal{R}^{v}, u_{i} \notin B^{v}, \ell+1 \leq i \leq \ell+m\right\}$, or $A$. 
Furthermore,

$$
|T|=\left|B^{v}\right|+\left(\gamma_{t 2}(G)-\left|\mathcal{R}^{v}\right|+|S|\right)=2\left|D^{v}\right|+\left(\gamma_{t 2}(G)-\left|\mathcal{R}^{v}\right|\right)<\gamma_{t 2}(G)
$$

which is a contradiction. (ㅁ)

Thus, by claim 1 ,

$$
N=\sum_{v \in V(H)}\left|\mathcal{R}^{v}\right| \leq \sum_{v \in V(H)} 2\left|D^{v}\right|=2|D|
$$

We now show a semi-total dominating set of $H$ in terms of $M_{i}$.

Claim 2 For any $i \in[k]$, there exists a set $X_{i}$ of at most $\left|R_{i}\right|-1$ vertices of $V(H)$ so that $M_{i} \cup P_{i} \cup X_{i}$ is a semi-total dominating set of $H$.

Proof. We first observe that $P_{i} \cup M_{i}$ is a dominating set of $H$ with the additional property that the vertices of $M_{i}$ dominate only themselves, not their neighbors. Thus, every vertex $x \in R_{i}$ must be either of distance 3 to some vertex $y \in R_{i}$ or every vertex of distance 2 from $x$ is a vertex of $M_{i}$. This holds since otherwise some vertex of distance 2 from $x$ is not dominated by $P_{i} \cup M_{i}$. Furthermore, if $x \in R_{i}$ which is of distance 3 to some vertex $y \in R_{i}$, then we may select one vertex $z$ on a path from $x$ to $y$ such that $z$ is of distance at most 2 to both $x$ and $y$.

We now construct a semi-total dominating set of $H, T_{i}$, by including the vertices of $M_{i}$, the vertices of $P_{i}$ and vertices $X_{i}$ which are of distance at most 2 to two vertices of $R_{i}$ which are themselves of distance three to each other. The minimum number of such vertices is at most $\left|R_{i}\right|-1$, which can be easily verified by induction on $\left|R_{i}\right|$, and the result follows. (ㅁ)

By Claim 2, for each $i$, we can construct a semi-total dominating set of $H, T_{i}=$ $M_{i} \cup R_{i} \cup Q_{i} \cup X_{i}$. This gives $\left|M_{i}\right|+\left|R_{i}\right| \geq \gamma_{t 2}(H)-\left|X_{i}\right|-\left|Q_{i}\right|$. However, note that $X_{i} \cap Q_{i}=\emptyset$ and $\left|X_{i}\right|+\left|Q_{i}\right| \leq\left|P_{i}\right|$. This implies that $\left|M_{i}\right|+\left|R_{i}\right| \geq \gamma_{t 2}(H)-\left|P_{i}\right|$. Thus, we have

$$
\sum_{i=1}^{k}\left(\left|M_{i}\right|+\left|R_{i}\right|\right) \geq \sum_{i=1}^{k}\left(\gamma_{t 2}(H)-\left|P_{i}\right|\right)=\gamma_{t 2}(G) \gamma_{t 2}(H)-|D|
$$

Combining equations (11), (3), and (4) we obtain

$$
|D| \geq \frac{1}{3} \gamma_{t 2}(G) \gamma_{t 2}(H)
$$




\section{Conclusion}

In this paper we have proven two Vizing's like results on the semi-total domination number. Our main result shown in Theorem 2 shows that for isolate-free graphs $G$ and $H$, it must be the case that $\gamma_{t 2}(G \square H) \geq \frac{1}{3} \gamma_{t 2}(G) \gamma_{t 2}(H)$. However, we do not believe this bound is sharp, and conjecture a stronger result.

Conjecture 1 For any isolate-free graphs $G$ and $H$,

$$
\gamma_{t 2}(G \square H) \geq \frac{1}{2} \gamma_{t 2}(G) \gamma_{t 2}(H) .
$$

\section{References}

[1] B. Brešar, Improving the Clark-Suen bound on the domination number of the Cartesian product of graphs, Discrete Math. 340 (2017) 2398-2401.

[2] B. Brešar, P. Dorbec, W. Goddard, B. Hartnell, M. Henning, S. Klavžar, D. Rall, Vizing's conjecture: a survey and recent results, J. Graph Theory, Vol. 69 (1) (2012) 46-76.

[3] B. Brešar, T.R. Hartinger, T. Kos, and M. Milanič, On total domination in the Cartesian product of graphs, manuscript.

[4] B. Brešar, M. A. Henning, D. F. Rall, Paired-domination of Cartesian products of graphs and rainbow domination, Electron. Notes Discrete Math. 22 (2005) 233-237.

[5] W.E. Clark, S. Suen An Inequality Related to Vizing's Conjecture, Electron. J. Combin. 7: N4 (2000).

[6] W. Goddard, M.A. Henning and C.A. McPillan, Semitotal domination in graphs, Util. Math. 94 (2014) 67-81.

[7] M.A. Henning, Edge Weighting Functions on Semitotal Dominating Sets Graphs and Combinatorics 33(2) (2017) 403-417.

[8] M.A. Henning and A.J. Marcon, On matching and semitotal domination in graphs, Discrete Math. 324 (2014) 13-18. doi:10.1016/j.disc.2014.01.021

[9] M.A. Henning and A.J. Marcon, Semitotal domination in graphs: Partition and algorithmic results, Util. Math., accepted

[10] M.A. Henning, A.J. Marcon, Vertices contained in all or in no minimum semitotal dominating set of a tree Discuss. Math. Graph Theory (2014)36(1), (2016) 71-93. 
[11] M.A. Henning, A.J. Marcon, Semitotal domination in claw-free cubic graphs Ann. Comb. (2016)20 (4) (2016) 799-813.

[12] M.A. Henning and D.F. Rall, On the total domination number of Cartesian products of graphs, Graphs Combin., 21 (2005) 63-69.

[13] M.A. Henning and A. Yeo, Total domination in graphs (Springer Monographs in Mathematics). ISBN-13: 978-1461465249 (2013).

[14] P. T. Ho, A note on the total domination number, Util. Math. 77 (2008) 97-100.

[15] Y. Lou and X. Hou, Total domination in the Cartesian product of a graph and $K_{2}$ or $C_{n}$, Util. Math. 83 (2010) 313-322.

[16] A.J. Marcon, Semitotal domination in graphs $\mathrm{PhD}$ dissertation, University of Johannesburg (2015)

[17] V.G. Vizing, The Cartesian product of graphs, Vycisl. Sistemy, 9 (1963) 30-43.

[18] Enqiang Zhu, Zehui Shao, and Jin Xu, Semitotal domination in claw-free cubic graphs, Graphs and Combinatorics (2017). https://doi.org/10.1007/s00373-017$1826-\mathrm{z}$ 